\title{
A granular resampling method based energy- efficient architecture for heartbeat classification in ECG
}

\author{
Yin Xu${ }^{1}$, Zhijian Chen ${ }^{1 a)}$, Xiaoyan Xiang ${ }^{2}$, and Jianyi Meng ${ }^{2}$ \\ ${ }^{1}$ Institute of VLSI Design, Zhejiang University, Hangzhou, China \\ ${ }^{2}$ State Key Laboratory of ASIC and System, Fudan University, Shanghai, China \\ a)chenzj@vlsi.zju.edu.cn
}

\begin{abstract}
SVM-based granular resampling method is put forward to obtain a robust classification model for energy-efficient ECG systems. The classification model consists of a low-complexity model to filter most easy-tolearn heartbeats and a high-accuracy classifier to identify the remained heartbeats. Energy-efficient hardware architecture for multi-class heartbeat classification is implemented based on the classification model. The architecture optimizations include memory segmentation to reduce energy consumption and time domain reuse to save resources. We adopt 40-nm CMOS process to implement the proposed design. It provides an average prediction speedup by $57.21 \%$ and a significant energy dissipation reduction by $52.22 \%$ per classification compared with the design without low-complexity models. Keywords: heartbeat classification, granular resampling method, energyefficient architecture, SVM
\end{abstract}

Classification: Integrated circuits

\section{References}

[1] S. Jain, et al.: "Efficient algorithm for classification of electrocardiogram beats based on artificial bee colony-based least-squares support vector machines classifier," Electron. Lett. 52 (2016) 1198 (DOI: 10.1049/el.2016.1171).

[2] S. Kiranyaz, et al:: "Real-time patient-specific ECG classification by 1-D convolutional neural networks," IEEE Trans. Biomed. Eng. 63 (2016) 664 (DOI: 10.1109/TBME.2015.2468589).

[3] N. Bayasi, et al.: "Low-power ECG-based processor for predicting ventricular arrhythmia," IEEE Trans. Very Large Scale Integr. (VLSI) Syst. 24 (2016) 1962 (DOI: 10.1109/TVLSI.2015.2475119).

[4] S.-Y. Hsu, et al.: "A 48.6-to-105.2 $\mu \mathrm{W}$ machine learning assisted cardiac sensor SOC for mobile healthcare applications," IEEE J. Solid-State Circuits 49 (2014) 801 (DOI: 10.1109/JSSC.2013.2297406).

[5] B.-Y. Shiu, et al:: "Low-power low-noise ECG acquisition system with dsp for heart disease identification," IEEE BioCAS (2013) (DOI: 10.1109/BioCAS. 2013.6679630).

[6] S. Jeong, et al.: “A $12 \mathrm{nW}$ always-on acoustic sensing and object recognition 
microsystem using frequency-domain feature extraction and SVM classification,” ISSCC (2017) 362 (DOI: 10.1109/ISSCC.2017.7870411).

[7] R. Batuwita and V. Palade: "FSVM-CIL: Fuzzy support vector machines for class imbalance learning," IEEE Trans. Fuzzy Syst. 18 (2010) 558 (DOI: 10. 1109/TFUZZ.2010.2042721).

[8] "Testing and reporting performance results of cardiac rhythm and ST segment measurement algorithms, ANSI/AAMI EC57:1998 standard," Association for the Advancement of Medical Instrumentation (1998).

[9] M. Llamedo, et al:: "Heartbeat classification using feature selection driven by database generalization criteria," IEEE Trans. Biomed. Eng. 58 (2011) 616 (DOI: 10.1109/TBME.2010.2068048).

[10] M. Galar, et al.: “An overview of ensemble methods for binary classifiers in multi-class problems: Experimental study on one-vs-one and one-vs-all schemes,” Pattern Recognit. 44 (2011) 1761 (DOI: 10.1016/j.patcog.2011.01. 017).

[11] MIT-BIH Arrhythmias Database http://www.physionet.org/physiobank/ database/mitdb/.

[12] C. Ye, et al.: "Heartbeat classification using morphological and dynamic features of ECG signals,” IEEE Trans. Biomed. Eng. 59 (2012) 2930 (DOI: 10. 1109/TBME.2012.2213253).

\section{Introduction}

Electrocardiogram (ECG), one of the important diagnostic methods for detecting heart diseases, records the time evolution of the heart electrical activity. In general, an ECG follows a standard morphological and rhythmic pattern. An indication of the abnormity is likely to be detected if the heart rate or rhythm or change in the morphological pattern happens [1]. Nowadays, automatic ECG interpretation based on machine learning techniques has made positive contributions to the timely detection and classification. However, it is challenging to establish a robust model for correct diagnosis, as external noise and between-class imbalance have influence on the classification performance [2]. Besides, designs with low-power consumption based on the robust model are essential for many ECG classification systems $[3,4]$.

To solve the problems aforementioned, there are several kinds of methods: obtaining low-noise signals with a specific analog frontend and extracting a unique set of ECG features to reduce effects of the noise and imbalance, using an easyimplementation classifier to satisfy low-power demands, such as naive bayes, maximum likelihood classification, linear support vector machine (SVM), and so on $[3,5,6]$. However, most methods in the literature are put forward to establish a robust model or obtain an energy-efficient implementation of the model. Few researches focus on both the two aspects mentioned simultaneously. In addition, most previous works aiming at low-power designs are interested in discriminating between normal and ventricular heartbeats and few works study multi-class heartbeat classification problems [3, 4].

In this letter, we propose a new SVM-based method called Granular Resam- 
obtain a robust classification model for energy-efficient ECG systems, which consists of a low-complexity model and a high-accuracy classifier. The lowcomplexity model, which is less computationally demanding, is used as a filter to identify most easy-to-learn heartbeats and the high-accuracy classifier is used to discriminate the remained heartbeats. An energy-efficient hardware design for three-class heartbeat classification problems is implemented based on the classification model according to one-vs-all (OVA) strategy. The architecture is optimized with memory segmentation and time domain reuse techniques to save energy and resources further.

\section{SVM-based granular resampling method}

Support vector machine (SVM) is a kernel-based supervised learning algorithm aiming at two-class problems and widely applied to many real-world classification problems in different domains [7]. The goal of the SVM learning algorithm is to find a hyperplane which has the maximum margin from the two classes and can separate the data samples of the two classes efficiently. To get the classification function of SVMs, informative samples called support vectors (SVs) need to be found. Besides, kernel functions are applied to satisfy the need of solving nonlinear classification problems. The commonly used kernel functions are linear, polynomial, and radial-basis function (RBF). In this letter, the RBF-based SVM, which is shown in Eq. (1)-(2), is chosen in terms of its strong applicability and generality.

$$
\begin{gathered}
K\left(x_{i}, x_{j}\right)=\exp \left(-\gamma\left\|\boldsymbol{x}_{\boldsymbol{i}}-\boldsymbol{x}_{\boldsymbol{j}}\right\|^{2}\right) \\
y=\sum_{i=1}^{n_{s v}} y_{i} \alpha_{i} K\left(\boldsymbol{x}_{\boldsymbol{i}}, \boldsymbol{x}\right)+b
\end{gathered}
$$

where $\alpha_{i}$ is the Lagrange multiplier, $y_{i}$ is the class label of a SV, $\mathbf{x}_{i}$ represents a SV, $\mathbf{x}$ is the input vector, $\mathrm{K}\left(\mathbf{x}_{i}, \mathbf{x}\right)$ is the kernel function, and $b$ is the bias.

The proposed SVM-based granular resampling method aims at two-class problems. In order to solve classification problems involving multiple classes, binarization strategy, one of the most popular techniques, is adopted in our method, which means multi-class problems will be transferred before they are processed. It is common to label one class with minor samples as a positive and the other one with major samples as a negative in a two-class problem. The classification problem can be well settled when a classifier which can separate samples of the two classes efficiently is obtained. However, the performance of classification models will be biased if training datasets are highly imbalanced or have noisy samples. Besides, most negative samples can be easily classified correctly by a low-complexity model instead of a high-accuracy classifier. Therefore, we adopt the granular resampling method to get a SVM-based model with the filter feature only using informative samples. Main idea of the proposed method is to use a weak classifier to extract informative samples from the negative class until the user defined condition is satisfied and potential noisy samples will be removed from informative samples in the first iteration. These extracted samples combined with all positive samples will be used as a new training dataset to obtain a high-accuracy 
classifier and a low-complexity model are obtained from informative samples extracted at the last iteration. Detailed steps are shown as follows:

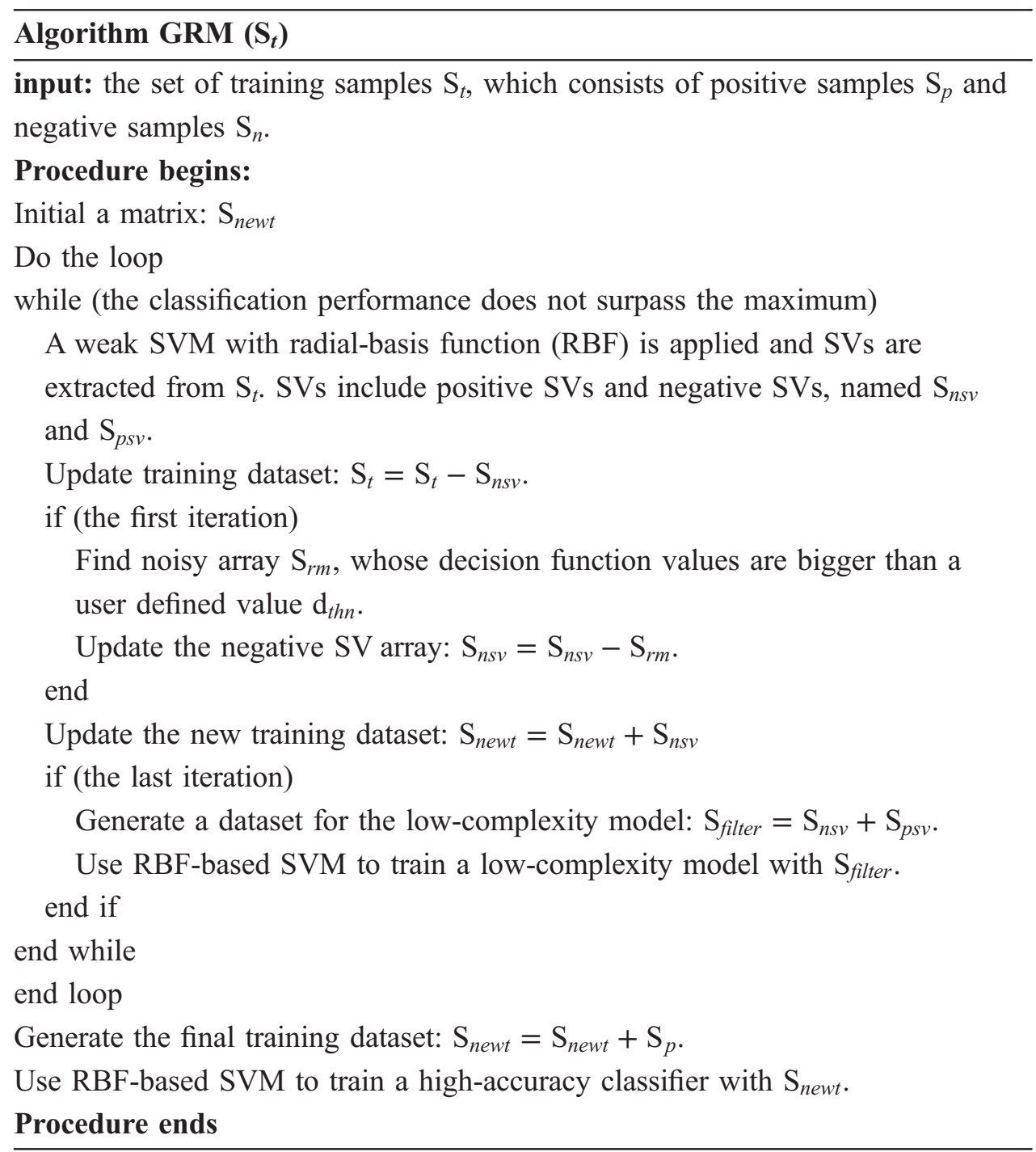

\section{Proposed hardware architecture}

Heartbeats, which have 16 classes, are re-clustered into five classes according to ANSI/AAMI EC57: 1998 standard [8]. However, not all classes are well represented in the database, hence a class-labeling modification is done in terms of [9] and the database is re-labeled as $\mathrm{N}$ class, $\mathrm{S}$ class, and $\mathrm{V}^{\prime}$ class. One-vs-one (OVO) and one-vs-all (OVA) are the most commonly-used strategies in the literature for multi-class classification [10]. Considering the resource consumption of a hardware implementation for three-class classification problems, OVA strategy is chosen in our design. The processing flow of three-class heartbeat classification is showed in Fig. 1. There are two stages to solve the problem and each stage consists of a low-complexity model and a high-accuracy classifier which are obtained from GRM. Most easy-to-learn heartbeats can be filtered by the model with low complexity and the remained hard-to-learn heartbeats will be classified by the classifier with high accuracy. 


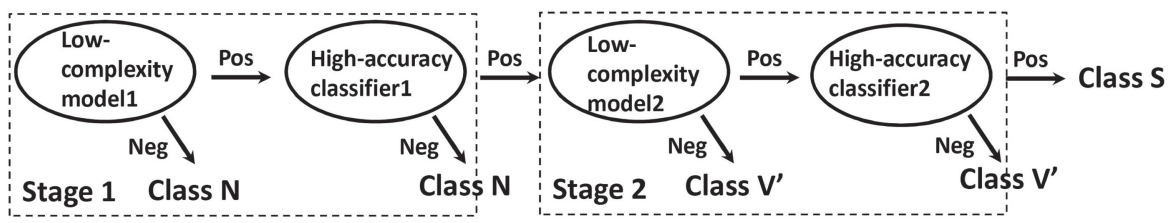

Fig. 1. The processing flow of the three-class classification.

Fig. 2 shows the corresponding recognition hardware architecture. As models and classifiers obtained from the proposed method have the same processing logic and only one model works at a time, implementing a SVM processing unit is enough and classifications can be completed by reusing the processing unit at a different time. Therefore, if an input cannot be identified, it will be returned to the input and reuse the processing unit with new parameters of the next model or classifier whose selection sequence is decided according to Fig. 1. The classification procedure of an input will not stop until it is identified before or by the highaccuracy classifier 2 . The parameters of models and classifiers are stored in the memory. However, heartbeat classification is a highly imbalanced problem and most heartbeats can be classified correctly by the model. Hence parameters of lowcomplexity models will be more constantly accessed than that of high-accuracy classifiers in the memory, resulting in a large amount of access energy dissipation. Therefore, we apply memory segmentation technique to reduce access energy efficiently. The parameter memory is divided into two sets and each set has two banks: one smaller memory storing the model and one large memory for the classifier. The smaller memories have a latch-based architecture, which is used to save access energy further.

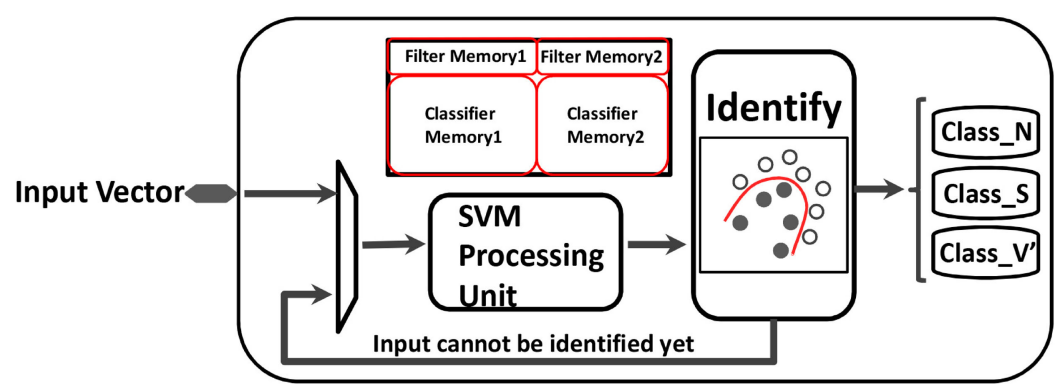

Fig. 2. The proposed recognition hardware architecture.

Fig. 3 shows the detailed implementation of the RBF-based SVM processing unit. The main computational units are an exponential function unit (EFU) and a parallel vector unit (PVU), which are described in detail as follows:

1) EFU: this unit is responsible for calculating $\exp (\mathrm{x})$. The exponential function is transferred and a new approximation can be represented as in Eq. (3) and Eq. (4).

$$
\begin{gathered}
\exp (x)=2^{\text {xlog } 2 e} \approx 2^{1.5 x} \\
2^{y}=2^{\text {floor }(y)+\text { delt }} \approx 2^{\text {floor }(y)}(\text { delt }+1)
\end{gathered}
$$

where $y$ is equal to $1.5 x$, floor $(y)$ means the largest integer not greater than $y$, and delt is the fractional part of $y$. The approximation will work well as all 
inputs are negative values. In order to implement the exponential approximation, a small area of hardware is occupied as only several adders and shifters will be used.

2) PVU: this unit is responsible for calculating $-\|\mathbf{x}-\mathbf{y}\|^{2} \times \gamma$, where $\mathbf{x}=$ $(\mathrm{x} 1, \mathrm{x} 2, \ldots, \mathrm{xn})$ represents a test vector, and $\mathbf{y}=(\mathrm{y} 1, \mathrm{y} 2, \ldots, \mathrm{yn})$ denotes a support vector. Norm squares are computed by multipliers and an adder tree is applied to sum these square values. As the parameter $\gamma$ is a power of two, remaining operations can be completed with a shifter and an adder to obtain the target value.

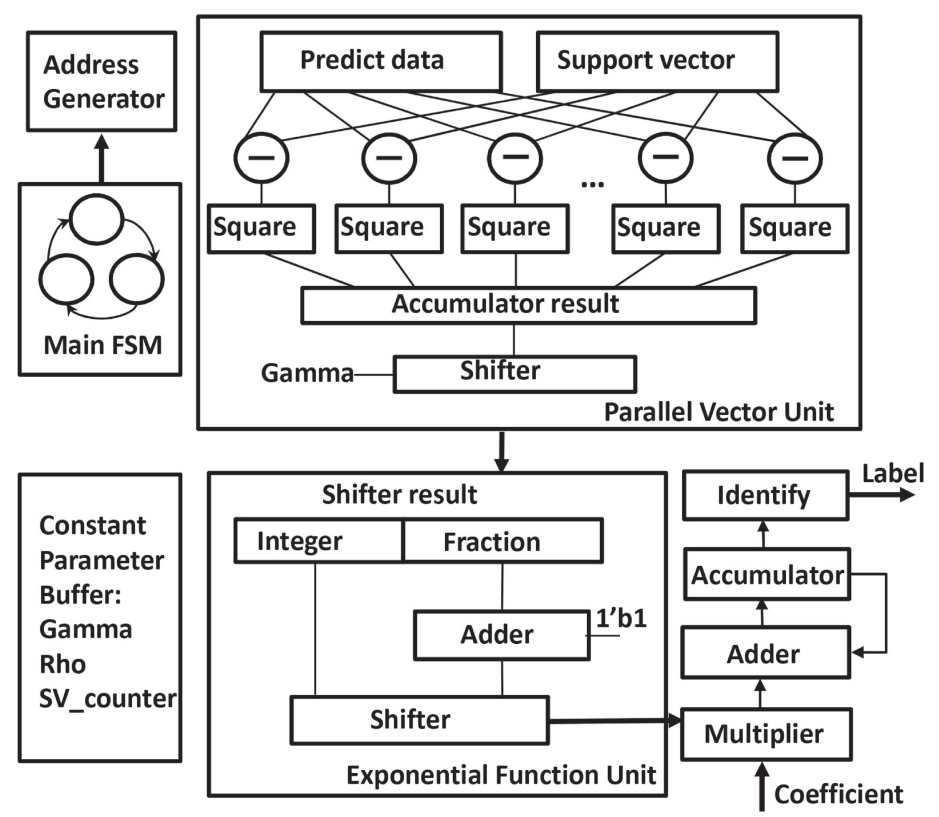

Fig. 3. The detailed implementation of the RBF-based SVM processing unit.

\section{Experiment}

To evaluate the performance of our classification model with filter features, the MIT-BIH arrhythmia database [11] is selected and it is a collection of 48 ECG recordings which are digitized at $360 \mathrm{~Hz}$. The four paced recordings are filtered out of database according to ANSI/AAMI EC57: 1998 standard [8]. In this letter, the remaining 44 records are divided following the database division in [12]. Heartbeat segments are obtained by segmenting these ECG signals using the annotations of QRS location. The two types of features, morphological-based features and timebased features, are extracted from ECG signals to represent heartbeat segments. Wavelet transform is used as a morphological feature extraction method as it provides characterizations in both temporal and frequency domains. Annotated $\mathrm{R}$ peak locations are used to form windows of ECG beats with length 109 samples and coefficients obtained from wavelet transform at level 4 within the window are extracted as the morphological features for each beat [12]. Time-based features including previous RR features and post RR features are obtained to characterize the dynamic information of the heartbeats. The previous RR feature is the interval 
Table I. The detailed results of the proposed method.

\begin{tabular}{ccccc}
\hline & \multicolumn{4}{c}{ Predicted Label } \\
\cline { 2 - 5 } Actual Label & N class & $\mathrm{V}^{\prime}$ class & $\mathrm{S}$ class & Total Number \\
\hline $\mathrm{N}$ & 42341 & 199 & 1637 & 44177 \\
\hline $\mathrm{V}^{\prime}$ & 157 & 2837 & 612 & 3606 \\
\hline $\mathrm{S}$ & 90 & 99 & 1645 & 1834 \\
\hline
\end{tabular}

Table II. The comparison results between the proposed method and three algorithms from the literature.

\begin{tabular}{|c|c|c|c|}
\hline Method & N class & S class & $V^{\prime}$ class \\
\hline$[12]$ & $88.64 \%(39157 / 44177)$ & $65.38 \%(1199 / 1834)$ & $74.88 \%(2700 / 3606)$ \\
\hline$[9]$ & $94.96 \%(41950 / 44177)$ & $77.54 \%(1422 / 1834)$ & $\mathbf{8 0 . 7 3 \% ( 2 9 1 1 / 3 6 0 6 )}$ \\
\hline$[2]$ & $81.21 \%(40963 / 50439)$ & $55.73 \%(1440 / 2584)$ & $72.85 \%(4744 / 6512)$ \\
\hline The proposed & $\mathbf{9 5 . 8 4 \% ( 4 2 3 4 1 / 4 4 1 7 7 )}$ & $\mathbf{8 9 . 6 9 \% ( 1 6 4 5 / 1 8 3 4 )}$ & $78.67 \%(2837 / 3606)$ \\
\hline
\end{tabular}

between a given $\mathrm{R}$ peak and its previous $\mathrm{R}$ peak, while the post $\mathrm{RR}$ feature is calculated between a given $\mathrm{R}$ peak and its following $\mathrm{R}$ peak. Besides, principal component analysis is applied to reduce the dimensionality of morphological-based features and balance the importance between the two types of features. Four principal components are selected to retain approximately $90 \%$ of the variance. Finally, each heartbeat segment is represented with six mixed features.

The classification model is trained based on GRM using the feature array extracted from the MIT-BIH arrhythmia database aforementioned. The classification performance and results compared with three state-of-art methods are listed in Table I and Table II. Results in the tables shows that our method can exhibit comparable performance with other methods.

The proposed recognition architecture based on the obtained classification model is designed in the Verilog HDL and synthesized using a commercial 40nm CMOS standard cell library. The on-chip memory is generated by the corresponding SRAM compiler in 40-nm process. Classification parameters of classifiers are stored in the generated SRAM and the size of SRAM is selected according to the bit-width and number of parameters. In our heartbeat classifiers, each support vector contains six features which can be represented with 16-bit fix-point and the corresponding Lagrange multiplier is represented with 32-bit fix-point. Hence the bit-width of a support vector combined with the corresponding coefficient is 128 . Besides, the support vector number of high-accuracy classifier 1 and high-accuracy classifier 2 is slightly less than 512 and 256, respectively. Therefore, SRAM with $12 \mathrm{kB}$ size is chosen to store the classification parameters. We follow the typical ASIC design flow to perform the logic synthesis, floor-plan, placement, and routing. Parasitic extraction is done after the layout generation. Three designs, architecture with latch-based low-complexity models, architecture with memorybased low-complexity models, and architecture without low-complexity models, are implemented to evaluate optimizations on energy consumption. Fig. 4 shows 
Table III. The comparison results of hardware implementation among three designs at $1 \mathrm{MHz}$.

\begin{tabular}{l|c|c|c|c|c|c}
\hline & \multicolumn{7}{c}{ Average Energy $(\mathrm{nJ})$} & \multicolumn{3}{c}{ Average Speed (cycle) } \\
\hline Design Type & $\mathrm{N}$ & $\mathrm{V}^{\prime}$ & $\mathrm{S}$ & $\mathrm{N}$ & $\mathrm{V}^{\prime}$ & $\mathrm{S}$ \\
\hline Latch-based architecture & 10.13 & 40.06 & 48.95 & 101.08 & 447.92 & 562.5 \\
\hline Memory-based architecture & 14.33 & 50.41 & 59.93 & 102.39 & 451.36 & 567 \\
\hline Without Filter & 29.94 & 41.21 & 41.21 & 349 & 512 & 512 \\
\hline Ratio of three classes in terms of predicting database: \\
N class : $\mathrm{V}^{\prime}$ class : S class $=85.83 \%: 6.32 \%: 7.85 \%$. \\
In the fist stage, 79.89\% samples from N class can be filtered correctly by the filter. \\
In the second stage, 70.51\% samples from $\mathrm{V}^{\prime}$ class can be filtered correctly by the filter. \\
Latch-based architecture: $\mathrm{A}_{\text {energy }}=15.07(\mathrm{~nJ}), \mathrm{A}_{\text {speed }}=159.22$ (cycle). \\
Memory-based architecture: $A_{\text {energy }}=20.19$ (nJ), $\mathrm{A}_{\text {speed }}=160.92$ (cycle). \\
Without Filter: $\mathrm{A}_{\text {energy }}=31.54$ (nJ), $\mathrm{A}_{\text {speed }}=372.09$ (cycle). \\
\hline
\end{tabular}

the energy consumption per classification for three designs at different frequency, where the energy consumption of different classes using low-complexity models is calculated in terms of Eq. (5). The maximum frequency of the proposed design is $100 \mathrm{MHz}$. From the figure, the higher frequency is, the less energy consumes for per classification. However, the advantage of energy saving brought by latch-based design becomes small gradually. Although operation frequency (sub-kHz) is enough for heartbeat classification processing, the frequency at $1 \mathrm{MHz}$ for both memory and processing unit is chosen as operating frequency in our design for energy consideration. Average energy and average speed, as the general criteria in this letter, are calculated according to Eq. (5)-(8) for performance comparison among different designs.

$$
\begin{aligned}
& E_{\text {per_class }}=P_{\text {filter }} \times F_{\text {energy }}+P_{\text {classifier }} \times C_{\text {energy }} \\
& A_{\text {energy }}=P_{N_{\text {_class }}} \times E_{N_{\text {_class }}}+P_{V^{\prime} \_ \text {class }} \times E_{V^{\prime} \_ \text {class }}+P_{S_{-} \text {class }} \times E_{S_{-} \text {class }} \\
& S_{\text {per_class }}=P_{\text {filter }} \times F_{\text {speed }}+P_{\text {classifier }} \times C_{\text {speed }} \\
& A_{\text {speed }}=P_{N_{-} \text {class }} \times S_{N_{\text {_class }}}+P_{V^{\prime} \_ \text {class }} \times S_{V^{\prime} \_ \text {class }}+P_{S_{-} \text {class }} \times S_{S_{-} \text {class }}
\end{aligned}
$$

where $\mathrm{E}_{\text {per_class }}$ and $\mathrm{S}_{\text {per_class }}$ represent energy consumption and classification speed per class, $\mathrm{A}_{\text {energy }}$ and $\mathrm{A}_{\text {speed }}$ mean average energy consumption and classification speed of the recognition architecture, $\mathrm{P}_{\text {filter }}$ and $\mathrm{P}_{\text {classifier }}$ are the percent of filtered samples and the remained separately, and $\mathrm{F}_{\text {energy }}$ and $\mathrm{C}_{\text {energy }}$ indicate the energy consumption of the model and the classifier, respectively. Detailed information about performance of hardware architecture is listed in the Table III, which shows our proposed architecture has advantages in the classification speed and energy consumption. Fig. 5 shows the layout along with a summary table, which shows latch-based memory and SRAM for classification parameters are area-consumption. Although latched-based memory for low-complexity models will increase area, it is beneficial for energy saving. 

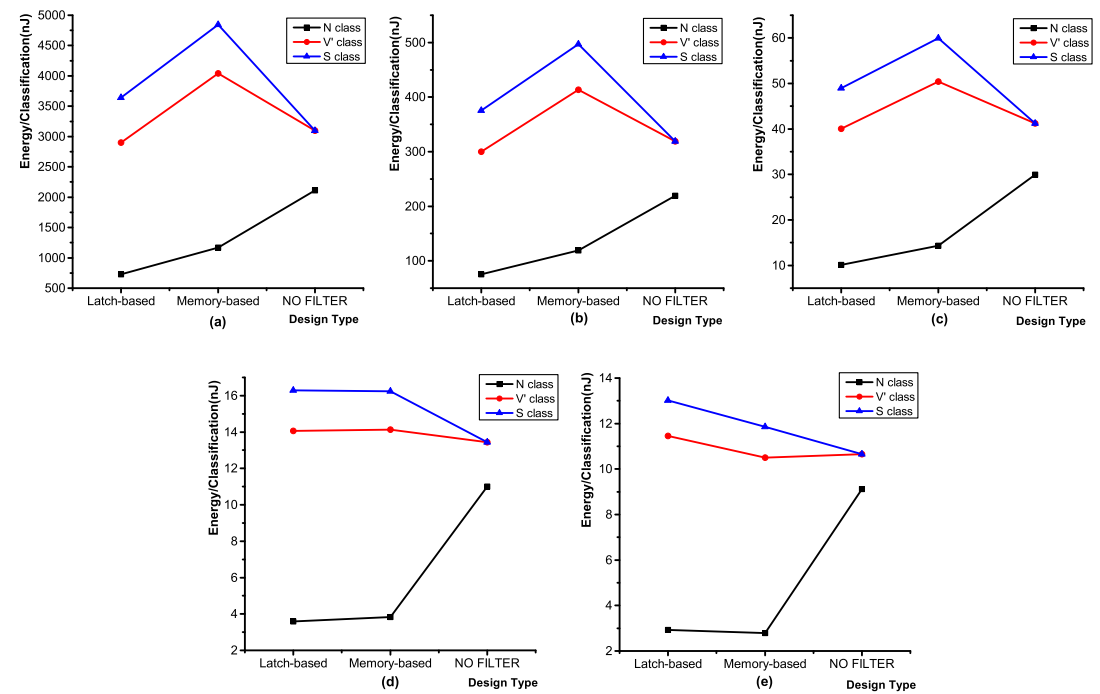

Fig. 4. The energy consumption according to various frequencies: (a) at $10 \mathrm{kHz}$, (b) at $100 \mathrm{kHz}$, (c) at $1 \mathrm{MHz}$, (d) at $10 \mathrm{MHz}$, (e) at $100 \mathrm{MHz}$.

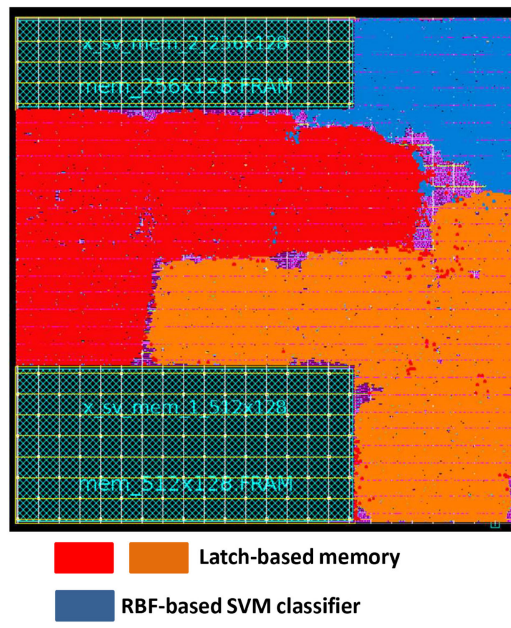

\begin{tabular}{|l|l|}
\hline Technology & SMIC $40 \mathrm{~nm}$ \\
\hline Supply Voltage & $1 \mathrm{~V}$ \\
\hline Max Frequency & $100 \mathrm{MHz}$ (Processing unit) \\
\hline Chip Area & $0.134 \mathrm{~mm}^{2}$ \\
\hline Gate Count & $129.2 \mathrm{k}$ \\
\hline Memory & $12 \mathrm{kB}$ (@1V,1MHz) \\
\hline $\begin{array}{l}\text { Energy } \\
\text { Consumption }\end{array}$ & $\begin{array}{l}10.13 \mathrm{~nJ} / \mathrm{N} \text { class sample } \\
40.06 \mathrm{~nJ} / \mathrm{V}^{\prime} \text { class sample } \\
48.95 \mathrm{n} / \mathrm{S} \text { class sample } \\
@ 1 \mathrm{~V}, 1 \mathrm{MHz}\end{array}$ \\
\hline
\end{tabular}

Fig. 5. The layout of proposed design and implementation details.

\section{Conclusion}

This letter proposes an energy-efficient architecture for heartbeat classification. A SVM-based granular resampling method is put forward to obtain a robust classification model which has a low-complexity model to filter most easy-to-learn heartbeats. We implement different kinds of hardware designs which include architecture with filter features and without filter features based on the obtained classification model for the performance comparison. Architecture optimizations including memory segmentation and time domain reuse are adopted to reduce energy consumption and resources, respectively. The designs are synthesized with a 40-nm CMOS technology and the entire layouts are generated for post-layout analysis. The proposed recognition design with filter features occupies $0.134 \mathrm{~mm}^{2}$ area and consumes average $15.07 \mathrm{~nJ} /$ classification at operating frequency of $1 \mathrm{MHz}$ and from an operating voltage of $1 \mathrm{~V}$. Results show that the design can save average energy and speed classification up by $52.22 \%$ and $57.21 \%$ per classification 
compared with non-filter-based architecture. In addition, smaller memories replaced by latch-based architecture reduce read energy of parameter memory by $25.36 \%$.

\section{Acknowledgments}

This work has been supported by the Fundamental Research Funds for the Central Universities (grant No. 2015QNA4018), and the State Key Laboratory of ASIC and System (grant No. 2015KF009). 